\title{
The Effect of Multilingualism on Cognition, Memory, and Emotions: A Review
}

\author{
Fotini Anastassiou, Ph.D. (Corresponding author) \\ Hellenic Open University \\ E-mail: fanastassiou@gmail.com
}

Received: December 3, 2020 Accepted: December 28, 2020 Published: December 31, 2020

doi:10.5296/ijld.v10i4.18134

URL: https://doi.org/10.5296/ijld.v10i4.18134

\begin{abstract}
A very popular field in research on bilingualism and multilingualism is how bilingual and multilingual people perceive and express their feelings and which languages they prefer each time (Pavlenko, 2012). A fascinating issue is that of distancing oneself from an L2 something that has also been discussed in the texts of bilingual writers who have stated that their L2 was for them a less emotionally charged language, in comparison with their L1. Besides, a speaker often uses his L1 to express emotions, since each language can convey distinct emotional meanings according to the interlocutors and the context.

Moreover, research on conscious event retrieval and autobiographical memory has also provided evidence for memory and language, as it has been found that memories tend to be more readily available for retrieval in the language in which they first appeared. Bilinguals generally report their memories in more detail and the level of processing during memory retrieval is higher in the language in which the event occurred. The exploration of the effect of multilingualism on memory and emotions is a promising field that can help educators and academics better understand the speakers' abilities and specific attributes.
\end{abstract}

Keywords: multilingualism, effect, cognition, memory, emotions, multilingual speakers

\section{Introduction}

According to Aronin (2005), the twentieth century can be distinguished into three stages as far as the study of the use of language is concerned. The first stage, which is defined as the monolingual, starts from the beginning of the twentieth century and goes up until the 1950s. The second stage, the bilingual one, extends from approximately the 1960s until the end of the 1980s, whereas the third stage, which is defined as the tri - multilingual, starts from the early 1990s continuing until today. Based on this classification, as well as the fact that bilingual speakers are learners of multiple language systems, we will consider anyone that is 
speaking more than one language as multilingual. Furthermore, anyone that has already acquired more than one language is more likely to proceed with learning a third or even a fourth language in the course of his/her life. A child's ability to communicate in more than one language is surely more complex and thus represents an intricate phenomenon too (Anastassiou, 2014). This phenomenon entails acquiring more than one grammatical system as well as language learning processes that are not specific to a single isolated system.

Researchers now agree and recognize that multilingualism has benefits and that it "brings opportunities not only to the individual but also to society as a whole" ( $\mathrm{Li}$, Dewaele, \& Housen, 2002: 3). This is a re-examination of the previous belief that bilingualism was a phenomenon that could cause psychological and social problems to the speakers. According to Edwards (2003), several alleged disorders were once associated with bilingualism stuttering, left-handedness, and even excessive materialism - noting that the majority of them are merely silly correlations and point to "xenophobic" social causes. Edwards points out that "when even emotional problems are associated with bilingualism, we have a classic example of the fallacy that correlation also implies causation" (p. 33).

Grin, Sfreddo, and Vaillancourt (2010) pinpointed the benefits that multilingualism has for the economic development of individuals and businesses. They focused on how multilingualism and variables in the economy affect each other. They used an econometric approach to examine the differences in corporate profits, which are based on language skills in the workplace and results showed that employers value their employees' language skills and pay them according to their qualifications. This kind of study is valuable as it can trigger policymakers to initiate policies that will promote multilingualism and will be of benefit to people and societies. Although official language policies of several states formally promote foreign language learning they do not promote the learning of minority languages in their country and if they are taught they are still not planned to reach high proficiency levels.

Besides, immigrant parents who have used their heritage languages with their children at home were suggested by education authorities to prefer the official language of the host country, to facilitate the social integration of children, and to facilitate their future academic success. The use of different languages at home is often - even today - seen as a cause of problems to the linguistic, social, and cognitive development of children (Anastassiou \& Andreou, 2014).

Baetens-Beardsmore (2003) suggested that conflicts of bilingualism are usually based on two basic types of fears: fears that reflect social concerns and fears that focus on the individual (although these two fears are often interrelated). He distinguishes between educational fears and political-ideological fears in societies. Educational fears are related to children's ability to deal with two languages in education. There is a difference between "elite bilingualism" on the one hand - resulting from a conscious decision made by middle-class parents, who can support the educational process with some, albeit small, involvement of their own. In contrast, there is transitional bilingual education, that the target is to integrate young students into monolingual education rather soon. Political-ideological fears are associated with a sense of danger to national identity. These worries are usually seen in traditionally monolingual 
societies, where bilinguals are sometimes perceived as individuals with two opposing identities and vague political beliefs. In essence, this fear involves the fear of the unknown (Dewaele, 2015).

The second category by Baetens-Beardsmore (2003) consists of cultural fears as parents may feel worried about the language and potential cognitive problems that the children may face, but also about a possible inability to be culturally committed to the culture of their heritage. These cultural fears are linked to identity conflicts, which could lead to marginalization and alienation within the community. Monolingual perspectives in a society are more likely to dominate in countries that the official language is considered a "language of wider communication". These views lead to linguistic "myopia", which is often connected with a low cultural awareness of the value of languages and is supported by government policies that exclusively provide for the official language (Edwards, 2003). Dewaele (2015), however, observes that the right tendency to introduce the foreign language from a very early age in schools (including kindergartens), leads to high proficiency, but is not the only key to success, as it has been shown by research how success is related to several other factors, like the power and quality of the language input, as well as the capability to know how to facilitate language usage in actual communication.

\section{The Effect of Multilingualism on Cognition}

A key question that has been the main issue of modern research on multilingualism is whether a person's linguistic experience, for example in a bilingual environment, affects his linguistic and cognitive development (Bialystok, 2001). Psycholinguists using behavioral study and neuro-imaging methods have conducted significant studies on the cognitive development of bilingual and multilingual individuals. Bilinguals are better than monolinguals in some non-verbal tasks that aid cognitive abilities, such as executive functions. Bilinguals appear to have better inhibitory control than monolinguals, and perform better in monitoring, switching, and information (Bialystok, Craik, \& Luk, 2012).

Bilingual cognitive advantages are traditionally attributed to an individual's knowledge of his or her two language systems and the need to interfere with a language when it is not required to emerge while speaking. Research shows that a third language may provide an additional cognitive advantage, as trilingual children showed better results than monolingual and bilingual children. Bialystok, Craik, \& Luk (2012) propose that the influence of bilingualism on cognition is more invisible in adults, but begin to play a more important role at an older age. They also argue that the use of cognitive control networks for bilingual processing can redefine and enhance them, promoting "mental flexibility", that is, the ability to adapt to constant change and efficient information processing. Thus, bilinguals are called upon to flexibly switch between their two language systems (Bialystok, 2001b), for example, when bilingual children talk to their parents, using with each parent his or her mother tongue or the one feeling more competent with (OPOL system).

Older bilinguals have a wider "cognitive reserve", which may be able to delay the onset of symptoms in people with dementia. It has been observed that the symptoms of dementia appear in bilinguals four years later compared to monolinguals (Bialystok., Craik, \& 
Freedman, 2007). Alladi et. al (2013) report that their research showed that the onset of Alzheimer's disease was 4.5 years later than monolinguals, and this finding was true of illiterate participants in the study, which the researchers believe shows that education is not necessarily a strong factor in delaying the onset of the disease. However, research in this area continues to provide findings that need further investigation, as more long-term studies with objective behavioral and neuroimaging measurements are needed since evidence suggests that delayed onset dementia may be related to bilingualism. However, we still do not have clear conclusions (van den Noort, et. al., 2019). Some researchers have wondered if this advantage could be linked to the existence of two distinct cultures in the minds of bilinguals, who usually own their distinct principles (Dewaele, 2015).

\section{The Effect of Multilingualism on Memory}

Research on conscious event retrieval and autobiographical memory has also provided evidence of memory and language, as it has been found that memories tend to be more readily available for retrieval in the language in which they first appeared. Bilinguals generally report their memories in more detail and the level of processing during memory retrieval is higher in the language in which the event occurred. A study of the autobiographical memory of bilingual immigrants, who changed their daily use of their language as a result of immigration, reinforced this view. This study looked at the period between the ages of about 10 and 30, which is associated with a higher number of autobiographical memories. The most interesting finding was that the participants' memories were separated between their two known languages. Memories before migration were stored in the original language of the participants. Memories from the post-migration period were later stored and retrieved more easily in the language in which participants were forced to turn as a result of migration (Schrauf \& Rubin, 1998, 2000, 2001). The processing of emotional speech by bilinguals has already begun to clarify some of the temperaments of semantic and autobiographical retrieval and structure of memory. Emotional stimuli can have the same impact regardless of language, as long as research participants have similar levels of fluency and daily use in all languages.

The surge of research on the influence of bilingualism and multilingualism on cognition contrasts with rather little interest in the effects of bilingualism and multilingualism on psychology matters. Dewaele and Stavans (2014) reproduced previous research conducted by the first author, in which knowledge of more languages was associated with higher scores in the areas of cultural empathy and open-mindedness and significantly lower scores in the area of emotional stability. Dewaele and Stavans reported that knowing more languages did not influence participants' personality ratings, but increased proficiency and frequency of usage of different languages were associated with significantly higher rates of cultural empathy and open-mindedness. Thus, it seems that knowledge of several languages and may create a person more oriented to the multicultural dimension of society, it expands thinking and people can be more aware of the random nature of their cultural values and more willing to accept that other people can have different ones.

Dewaele and Li (2013: 237) investigated the relationship between multilingualism and 
ambiguity tolerance (AT) in more than 1,500 monolingual, bilingual, and multilingual participants. Monolinguals and bilinguals gave scores that were significantly lower on this test compared to multilinguals. Participants with higher levels of multilingualism, and especially those who had lived abroad, also had a greater tendency to tolerate ambiguity. The researchers concluded that the social, linguistic, and cultural environment strongly reinforces this value - although the magnitude of this effect is small. So far studies have also pointed out that speakers who know more languages usually report less communication stress, and this includes communication in their L1 (Dewaele, 2013). This reduced communication stress has been associated with multilinguals' experience in communicating with a variety of interlocutors, which helps them to get through any potential problems. Therefore, the presence of multilingualism in a person's environment may contribute to the formation of his personality (Dewaele, 2015).

\section{Communicating Emotions: Multilinguals' Language Choices}

A very popular field in research on bilingualism and multilingualism is how bilingual and multilingual people perceive and express their feelings and which languages they prefer each time (Pavlenko, 2012). A fascinating issue is that of distancing oneself from his L2, which has also been seen in the texts of bilingual writers, like Nancy Huston (L1 English, L2 French), who stated that French was for her a less emotionally charged language, in comparison with her L1 (English). She lived in Paris for many years, and when she gave an interview on French radio about language preferences for expressing strong emotions, such as sudden anxiety, she replied that English was her preferred language. The journalist then asked her what she would say in such a case and the author answered using English, but at the end of her sentence, she also used French swear words. She was surprised when she realized it (Dewaele, 2013: 131). Her surprise because of the sudden emergence of the French swear word was obvious and she seemed to understand that her language choices were changing and that French had become more solid in the emotional world and mental lexicon.

Pavlenko (2012) argues that L1 is usually the language used to express emotions, since each language a multilingual owns may have distinct emotional connotations, and this depends on the interlocutors and the context. She also adds that the emotional processing in L1 is more automatic and that multilinguals show an increased electrodermal reaction to words that express emotions. Due to the lower levels of automation in emotional processing in L2, there is less interference and less electrodermal reaction to negative emotional stimuli. According to Pavlenko, some bilinguals do not acquire a second language when they are children and there are foreign language users who learn it at an older age; in these cases, their languages may be integrated differently, and processed in a semantic rather than emotional way.

Dewaele (2013) investigated the influence of three groups of independent variables - the linguistic history of bilingual and multilingual, the current use of language, social biography, and psychological variables - in language perception and language choice for communicating different emotions and for foreign language stress. Several tendencies appeared in the dataset where more than 1,500 participants and 20 multilinguals were interviewed about their languages and their usage. The frequent appearance of an LX to express emotions, the 
positive views about an LX, and the low levels of stress for the foreign language are associated with the young age of acquiring an LX, the natural way of learning, or the mixed learning of LX (and not only through formal teaching), the frequent usage of LX, the higher usage of the LX in social environments and the greater range of interlocutors. The self-report on the degree of code-switching frequency was also found to be quite higher when participants talked about topics that were more emotionally charged with known interlocutors, compared to discussing topics that were not associated with particular emotions with interlocutors that were new to the speaker.

Swearing and the phrase "I love you" were found to be significantly emotionally stronger in the L1 of multilinguals, particularly when they were more frequently used. "Indeed, for some multilingual groups, more often for Asians and Arabs, the use of specific words or expressions with strong emotional repercussions was taboo in their own culture. However, it is important not to overgeneralize, as other types of strong emotional language can be used, especially negative terms or language that evokes feelings of fear. In the case of insults, the use of an LX allowed participants to overcome any social constraints. The analysis of language preferences and perceptions showed that the values and practices of the L1 culture remain so ingrained in these multilinguals that the corresponding words in the L1 are usually characterized by more emotion than the corresponding words in the LX (Dewaele, 2013: 15).

However, there seem to be cases of mixing the values and practices of L1 and LX. Although multilinguals can separate their languages during their communications there is a greater receptiveness of the two languages in a factual, non-verbal, and social area. Emotional socialization in LX leads to a special linguistic behavior in both L1 and LX - a good case of multi-competence (Cook \& Bassetti, 2010). The profanities in LX reflect the new liberty of expression, with no violation of the standards and habits of L1 and the special weight of its culture. Dewaele (2013: 17) states that "further research has shown that immigrants' memories of what they experienced in L1 are generally richer in terms of the emotional weight we give when we remember them in L1. When these memories are recalled in an LX, some of the emotional intensity is lost. This may not always be a bad thing, especially if the multilingual person is talking about traumatic events. This finding has important psychotherapeutic implications for the patient and the therapist". Costa and Dewaele (2014) surveyed psychotherapists who seemed to agree that knowledge of a foreign language helped them to collaborate better with their multilingual patients during their sessions. Even though no psychotherapist had tried to trigger or "allow" the usage of other languages during treatment other than the official language of the country, many were interested in the possibilities it might have. The number of multilinguals who have access to therapeutic services and who become therapists themselves in the future is constantly increasing, so a review of psychotherapy curricula may be helpful to take into account changing language needs and patient profiles but also of their doctors.

\section{Conclusion}

As time passes more and more research is being conducted on multilingualism and its various facades. The increasing interest in multilingualism is walking side by side with our era. 
People immigrate, communicate with other cultures, there are cases that countries have more than one official language and in general, we need to speak more than our mother tongue to be able to adapt to every aspect of our lives. Moreover, the European Union has proposed the "Mother tongue plus one" guideline suggesting that European citizens should learn apart from their mother tongue, one neighbor country's language, and another lingua franca. Research on multilingualism is demanding both because of the complexity that results from different interdisciplinary fields and because of the number of variables involved in it. Although a more realistic view of multilingualism seems to have been established, as a rule, the monolingual criterion, especially about proficiency, still prevails in several methodologies used in bilingualism and multilingualism studies. Future research on multilingualism will give more data on the way languages interact, how speakers subconsciously choose the languages they use to express their emotions, how their languages emerge when they recollect things, how they code-switch as well as more insights into multilingual speech production, including its related issues of language choice, language dominance, and directionality of switches.

\section{References}

Alladi, S., Bak, T. H., Duggirala, V., Surampudi, B., Shailaja, M., Shukla, A.K., ... Kaul, S., (2013). Bilingualism delays age at onset of dementia, independent of education and $\begin{array}{llll}\text { immigration } & \text { status. } & \text { Neurology. } & 81,\end{array}$ https://doi.org/10.1212/01.wnl.0000436620.33155.a4

Anastassiou, F. (2014). Children learning English as a third language (L3): A study on their L3 speech production (Unpublished doctoral dissertation. University of Thessaly, Greece).

Anastassiou, F., \& Andreou, G. (2014). Multilingualism in Greece: A study of speech production of trilingual children. (vol. 2 pp. 187-198). In Major Trends in Theoretical and Applied Linguistics: Selected Papers from the 20th International Symposium on Theoretical and Applied Linguistics. London: Versita de Gruyter. https://doi.org/10.2478/9788376560885.p22

Aronin, L. (2005) Theoretical perspectives of trilingual education. International Journal of Sociology of Language, 171, 7-22. https://doi.org/10.1515/ijsl.2005.2005.171.7

Baetens Beardsmore, H. (2003). Who is afraid of bilingualism? In J.-M. Dewaele, A. Housen, \& W. Li (Eds.), Bilingualism: Basic principles and beyond (pp. 10-27). Clevedon, UK: Multilingual Matters. https://doi.org/10.21832/9781853596315-004

Bialystok, E. (2001). Metalinguistic aspects of bilingual processing. Annual Review of Applied Linguistics, 21, 168-181. https://doi.org/10.1017/S0267190501000101

Bialystok, E. (2001b). Bilingualism in development: Language, literacy, and cognition. New York: Cambridge University Press. https://doi.org/10.1017/CBO9780511605963

Bialystok, E., Craik, F. I., \& Freedman, M. (2007). Bilingualism as a protection against the onset of symptoms of dementia. Neuropsychologia, 45, 459-464. https://doi.org/10.1016/j.neuropsychologia.2006.10.009 
Bialystok, E., Craik, F. I., \& Luk, G. (2012). Bilingualism: consequences for mind and brain. Trends in Cognitive Sciences, 16(4), 240-250. https://doi.org/10.1016/j.tics.2012.03.001

Cook, V. J., \& Bassetti, B. (2010). Language and bilingual cognition. New York, NY: Psychology Press. https://doi.org/10.4324/9780203836859

Costa, B., \& Dewaele, J.-M. (2014). Psychotherapy across languages: Beliefs, attitudes and practices of monolingual and multilingual therapists with their multilingual patients. Counseling and Psychotherapy Research, 14, 235-244. https://doi.org/10.1080/14733145.2013.838338

Dewaele, J.-M. (2013). Emotions in multiple languages (2nd ed.). Basingstoke, UK: Palgrave Macmillan.

Dewaele, J. -M. (2015). Bilingualism and Multilingualism. In K. Tracy, C. Ilie, \& T. Sandel (Eds), The International Encyclopedia of Language and Social Interaction, First Edition. Oxford, UK: John Wiley \& Sons. https://doi.org/10.5860/CHOICE.194235

Dewaele, J.-M., \& Li, W. (2013). Is multilingualism linked to a higher tolerance of ambiguity? Bilingualism: Language and Cognition, 16, 231-240. https://doi.org/10.1017/S1366728912000570

Dewaele, J. M., \& Stavans, A. (2014). The effect of immigration, acculturation and multicompetence on personality profiles of Israeli multilinguals. International Journal of Bilingualism, 18(3), 203-221. https://doi.org/10.1177/1367006912439941

Edwards, J. (2003). The importance of being bilingual. In J.-M. Dewaele, A. Housen, \& Li Wei (Eds.), Bilingualism: Basic principles and beyond (pp. 28-42). Clevedon, UK: Multilingual Matters. https://doi.org/10.21832/9781853596315-005

Grin, F., Sfreddo, C., \& Vaillancourt, F. (2010). The economics of the multilingual workplace. New York, NY: Routledge.

Li, W., Dewaele, J.-M., \& Housen, A. (2002). Introduction: Opportunities and challenges of bilingualism. In W. Li, J.-M. Dewaele, \& A. Housen (Eds.), Opportunities and challenges of bilingualism (pp. 1-14). Berlin, Germany: Mouton de Gruyter. https://doi.org/10.21832/9781853596315-003

Pavlenko, A. (2012). Affective processing in bilingual speakers: Disembodied cognition? $\begin{array}{llll}\text { International Journal of } & \text { Psychology, 47(6), }\end{array}$ https://doi.org/10.1080/00207594.2012.743665

Schrauf, R. W., \& Rubin, D. C. (1998). Bilingual autobiographical memory in older adult immigrants: A test of cognitive explanations of the reminiscence bump and the linguistic encoding of memories. Journal of Memory and Language, 39(3), 437-457. https://doi.org/10.1006/jmla.1998.2585

Schrauf, R.W., Rubin, D.C. (2000). Internal languages of retrieval: The bilingual encoding of memories for the personal past. Memory and Cognition, 28, 616-623. 


\section{Macrothink}

International Journal of Learning and Development

https://doi.org/10.3758/BF03201251

Schrauf, R. W., \& Rubin, D. C. (2001). Effects of voluntary immigration on the distribution of autobiographical memory over the lifespan. Applied Cognitive Psychology, 15, 75-88. https://doi.org/10.1002/acp.835

van den Noort, M., Vermeire, K., Bosch, P., Staudte, H., Krajenbrink, T., Jaswetz, L., ... Lim, S. (2019). A Systematic Review on the Possible Relationship Between Bilingualism, Cognitive Decline, and the Onset of Dementia. Behavioral sciences (Basel, Switzerland), 9(7), 81. https://doi.org/10.3390/bs9070081

\section{Copyright Disclaimer}

Copyright for this article is retained by the author(s), with first publication rights granted to the journal.

This is an open-access article distributed under the terms and conditions of the Creative Commons Attribution license (http://creativecommons.org/licenses/by/4.0/). 